\title{
Experimental infection in tumor necrosis factor alpha receptor, interferon gamma and interleukin 4 deficient mice by pathogenic Leptospira interrogans
}

\author{
Daniel A. Athanazio ${ }^{\mathrm{a}, \mathrm{b}}$, Cleiton S. Santos ${ }^{\mathrm{a}}$, Andréia C. Santos ${ }^{\mathrm{a}}$, \\ Flávia W.C. McBride ${ }^{a}$, Mitermayer G. Reis ${ }^{a}, b, *$ \\ ${ }^{a}$ Gonçalo Moniz Research Centre, Oswaldo Cruz Foundation, Ministry of Health, Salvador, Brazil \\ ${ }^{\mathrm{b}}$ Federal University of Bahia, Salvador, Bahia, Brazil
}

Received 26 August 2007; received in revised form 13 September 2007; accepted 20 September 2007

Available online 29 September 2007

\begin{abstract}
Only recently, knockout mouse models were applied in studies on the pathogenesis of leptospirosis. Current data suggest an important role of innate immunity receptors and interferon $\gamma$ dependant cellular response on protection. It is not clear, however, whether T helper cell polarization influences on outcome of leptospiral infection. We report findings of experimental infection of C57BL/6 (interferon $\gamma$ or tumor necrosis factor $\alpha$ receptor deficient) and BALB/c (interleukin 4 deficient) mice infected by pathogenic Leptospira interrogans serovar Copenhageni. Specific cytokine gene deficiency had no impact on outcome since all animals survived. TNFR knockout mice, however, exhibited more severe residual renal inflammation during convalescence thus suggesting this cytokine is important in early control of infection, protecting kidneys from relevant pathology.
\end{abstract}

(C) 2007 Elsevier B.V. All rights reserved.

Keywords: Leptospirosis; Leptospira; Mice; Knockout; Cytokines; Animal disease models

\section{Introduction}

Leptospirosis is a widespread zoonosis with broad clinical spectrum including fatal outcomes due to acute renal failure and pulmonary hemorrhage. Pathogenic leptospires are carried by diverse mammalian reservoirs and peridomiciliary rodents are the most important source of infection in urban settings. Despite multiple efforts to develop new vaccine strategies with longlasting effect and cross-protection among different serovars, our current knowledge about the immune determinants of host protection and pathogenesis is still elusive (McBride et al., 2005).

Diverse experimental animal models are used in leptospirosis research. Guinea pigs and hamsters are the most suitable laboratory rodents for reproduction of lethal acute infection while rats represent a prototype of resistance to acute disease and a potential model to study persistent infection (Nally et al., 2005a).

\footnotetext{
* Corresponding author at: Fundação Oswaldo Cruz (FIOCRUZ), Rua Waldemar Falcão, 121 Candeal, CEP 40296-710, Salvador, Bahia, Brazil. Tel.: +55 713176 2200; fax: +55 7131762326 .

E-mail address: miter@bahia.fiocruz.br (M.G. Reis).
}

Mice are recognized as resistant hosts usually developing persistent asymptomatic carrier state although this was not deeply evaluated in the diverse variety of mouse strains. Swiss mice may succumb or develop subclinical renal colonization as a function of age and inoculum size (Faine, 1962). Studies on cyclophosphamide-treated BALB/c mice suggested, based on experiments of passive immunization and transference of splenic B-cell subset, that humoral immunity is the key issue in natural resistance to disease. Athymic nude mice had no difference in susceptibility when compared to wild-type animals (Adler and Faine, 1977). The dogma regarding the major role of humoral response has been challenged by recent reports, showing a strong association between whole cell vaccine immunoprotection against serovar Hardjo among cattle and laboratory markers of cellular immunity activation, such as high interferon $\gamma($ IFN$\gamma$ ) secretion by peripheral blood mononuclear cells (PBMC) and rising of TCR $\gamma \delta+$ subset in peripheral blood (Brown et al., 2003; Naiman et al., 2002).

Transgenic or mutant murine models have only recently been applied to study leptospirosis and most data to date explore the role of innate immunity. Toll-like receptor 4 (TLR4) defective 
$\mathrm{C} 3 \mathrm{H} / \mathrm{HeJ}$ mice has already been used in studies on pathogenesis and immunoprotection assays to evaluate recombinant protein vaccine candidates (Koizumi and Watanabe, 2004; Nally et al., 2005b; Pereira et al., 1998), but only a recent report could attribute the higher susceptibility of this strain to leptospiral lethal infection to the absence of TLR 4 function, by using appropriated control strain with the same genetic background (Viriyakosol et al., 2006). TLR2 defective C56BL/6 mice are resistant to lethal effects of leptospiral lipopolysaccharide (LPS); however, no challenge experiments with live leptospires were performed to evaluate its role in host innate protection (Werts et al., 2001). Furthermore, severe combined immunodeficiency (SCID) $\mathrm{C} 3 \mathrm{H}$ mice are highly susceptible to lethal infection (Nally et al., 2005b; Viriyakosol et al., 2006). We conducted experiments in tumor necrosis factor $\alpha$ (TNF- $\alpha)$ receptor Rp55 (TNFR), interferon $\gamma$ (IFN- $\gamma$ ) and interleukin 4 (IL-4) knockout mouse models in order to evaluate the role of each branch of immunity on protection and pathogenesis of the most common target organ lesions in leptospirosis.

\section{Materials and methods}

\subsection{Bacteria}

Leptospira organisms were cultivated in liquid EllinghausenMcCullough-Johnson-Harris (EMJH) medium (Difco Laboratories, Detroit, MI) at $29{ }^{\circ} \mathrm{C}$ and counted in a Petroff-Hausser counting chamber (Fisher Scientific, Pittsburgh, PA). A clinical isolate from Brazil, Leptospira interrogans serovar Copenhageni strain FIOCRUZ L1-130 was used in all assays (Ko et al., 1999). This strain was passaged and re-isolated from hamsters four times, after isolation from a blood culture of a patient with leptospirosis and stored at $-70^{\circ} \mathrm{C}$. Frozen aliquots were thawed and passaged in liquid medium for seven times prior to use as a low-passage-number isolate in the infection experiments. We have previously studied the virulence of this strain at the same passage in the hamster model and the lethal dose 50\% was 46 leptospires (Silva et al., 2007).

\subsection{Study design}

All infected animals received intraperitoneal lower inoculum $\left(1.0 \times 10^{7}\right)$ or higher inoculum $\left(2.0 \times 10^{8}\right)$. The groups were assigned as follows:

- C57BL/6 TNFR-KO uninfected controls $(n=2)$

- C57BL/6 TNFR-KO infected with $1.0 \times 10^{7}$ inoculum $(n=5)$

- C57BL/6 TNFR-KO infected with $2.0 \times 10^{8}$ inoculum $(n=4)$

- C57BL/6 IFN-KO uninfected controls $(n=2)$

- C57BL/6 IFN-KO infected with $1.0 \times 10^{7}$ inoculum $(n=4)$

- C57BL/6 IFN-KO infected with $2.0 \times 10^{8}$ inoculum $(n=4)$

- C57BL/6 wild-type uninfected controls $(n=4)$

- C57BL/6 wild-type infected with $1.0 \times 10^{7}$ inoculum $(n=7)$

- C57BL/6 wild-type infected with $2.0 \times 10^{8}$ inoculum $(n=8)$

- BALB/c IL-4/KO uninfected controls $(n=2)$

- BALB/c wild-type uninfected controls $(n=2)$
- $\mathrm{BALB} / \mathrm{c}$ IL-4/KO infected with $1.0 \times 10^{7}$ inoculum $(n=4)$

- BALB/c wild-type infected with $1.0 \times 10^{7}$ inoculum $(n=4)$.

\subsection{Light microscopy}

Necropsies were performed immediately after sacrifice. Sacrifice was projected for animals presenting moribund state or for convalescent survivors after 28 days of infection. Kidney, lung, heart and liver were fixed in formalin $4 \%$, embedded in paraffin, cut 4-5 $\mu \mathrm{m}$ thick and used for conventional histology. The semi-quantitative analysis of the lesions was performed using the following criteria: $(+)$ mild when the lesion is seen less then once per $40 \times$ field; (++) 1-2 lesions per $40 \times$ field, and (+++) more than two lesions by $40 \times$ field. Specifically for quantification of interstitial nephritis, the criteria was complemented by the following: (+) infiltrates rich in macrophages and lymphocytes restricted to periarterial areas; (++) such infiltrates extending to other renal parenchymal zones with 1-2 lesions per $40 \times$ field; and (+++) for the same lesions detected in more than two areas per $40 \times$ field. Paraffin sections were also stained with Warthin-Starry standard technique (Faine, 1965).

\subsection{Statistics}

Comparison of proportions of categorical data was calculated by Fischer's exact test using Graph-Pad Prism 4.03 software package (Graph Pad, CA, USA).

\section{Results and discussion}

All mice survived with no signs of disease. Necropsies performed at 28-day after infection revealed aggregates of leptospires in renal tubules by Warthin-Starry stain (Fig. 1) in all infected animals, except for one animal from IFN-KO and other from C57BL/6 wild-type groups. Spirochetes were not identified in other tissue samples. The only lesion detected by light microscopy was renal inflammation and no pathology was observed in other tissues (Fig. 2). As shown in Table 1, C57BL/6 mice infected with lower inoculum exhibited interstitial nephritis grade ++ in 4/5 (80\%), 1/4 (25\%) and 2/7 (29\%) in TNFR-KO, IFN-KO and wild-type strains, respectively. Among higher inoculum infected C57BL/6 mice, the same lesion was detected in 3/4 (75\%) of TNFR-KO animals and in no IFN-KO or wild-type strains. The highest grade of renal inflammation was not observed in any case. When grouped together (both higher and lower inocula), TNFR-KO mice exhibited renal inflammatory lesions grade ++ more frequently than wild-type counterparts $(7 / 9$ or $78 \%$ versus $2 / 15$ or $13 \%, p<0.05)$. There was also a trend for IFN-KO mice to show absence of any degree of inflammation (Table 2) more frequently. All uninfected control groups exhibited no pathology. BALB/c mice, regardless of IL-4 gene status, survived without showing any pathology at day 28 .

Some experimental data based on transgenic models is consistent with concept that $\mathrm{T}$ helper polarization may affect 

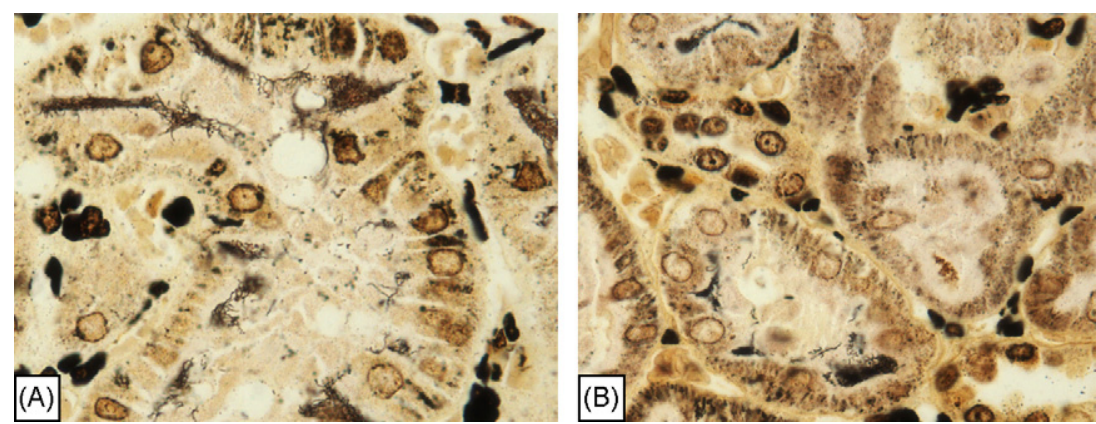

Fig. 1. Aggregation of leptospires in renal tubules 28 days after infection (Warthin-Starry, 2000×). A is a photomicrograph of a TNFR-KO animal and B is from a C56BL/6 wild-type mouse.

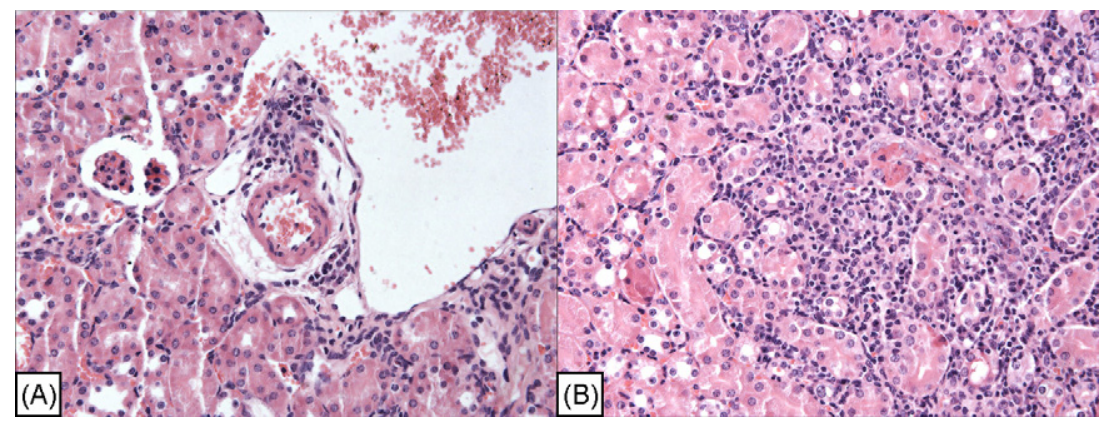

Fig. 2. Inflammatory infiltrates rich in lymphocytes and macrophages. A illustrates a periarterial lesion typical of grade $+($ hematoxylin-eosin, $400 \times)$ and B shows a more extensive infiltrate in cortical parenchyma typical of grade ++ (hematoxylin-eosin, $400 \times$ ).

Table 1

Interstitial nephritis grade ++ at 28-day after infection

\begin{tabular}{lll}
\hline Mouse strain & Inoculum $10^{7}$ & Inoculum $10^{8}$ \\
\hline C57BL/6 TNFR-KO & $4 / 5$ & $3 / 4$ \\
C57BL/6 IFN-KO & $1 / 4$ & $0 / 4$ \\
C57BL/6 wild-type & $2 / 7$ & $0 / 8$ \\
BALB/c IL4-KO & $0 / 4$ & \\
BALB/c wild-type & $0 / 4$ & \\
\hline
\end{tabular}

disease outcome. For instance, IL-4 defective mice show reduced parasitism and more severe inflammatory involvement of myocardium after infection by Trypanosoma cruzi (Soares et al., 2001). Conversely, the genetic deficiency of IL-4 or IFN$\gamma$ has little effect in experimental arthritis by the spirochete Borrelia burdorferi (Brown and Reiner, 1999; Satoskar et al., 2000).

In contrast to the mouse model of toll-like receptor 4 deficiency (Viriyakosol et al., 2006), the specific gene deficiency of

Table 2

Inflammatory lesions in infected animals at 28-day after infection in combined analysis of lower and higher inocula

\begin{tabular}{llll}
\hline Mouse strain & Nephritis ++ & Nephritis + & None \\
\hline C57BL/6 TNFR-KO & $7 / 9$ & $1 / 9$ & $1 / 9$ \\
C57BL/6 IFN-KO & $1 / 8$ & $2 / 8$ & $5 / 8$ \\
C57BL/6 wild-type & $2 / 15$ & $8 / 15$ & $5 / 15$ \\
BALB/c IL4-KO & & & $4 / 4$ \\
BALB/c wild-type & & & $4 / 4$ \\
\hline
\end{tabular}

each cytokine pathway studied exhibited little influence on infection outcome. The observation of more severe residual lesions in TNF- $\alpha$ receptor deficient mice indicates that this cytokine may have an important role, albeit partial, in early infection preventing wider dissemination and more severe acute lesions. IFN- $\gamma$ deficiency was not required for natural resistance to disease in our model and it indeed may prevent inflammatory residual lesions. This observation is relevant for future investigation since some late complications of leptospirosis, such as uveitis, has been attributed to cell-mediated immunopathogenesis (Rathinam, 2002).

The investigation on IL-4 influence on pathogenesis was hampered by the fact that BALB/c mice seems to be more resistant to leptospira-induced pathology than C57BL/6 mice. Variations on susceptibility to disease among mouse strains have not been systematically tested in leptospirosis and this restricts the selection and broader application of transgenic animal models. Our results suggest that C57BL/6 strain is more suitable for use in transgenic mouse models since wild-type animals may exhibit a second endpoint other than lethal outcome that can be easily measured (interstitial nephritis). The influence of humoral response on natural resistance should be better evaluated in other mouse strains such as C57BL/6 or in B-cell depleted strains.

\section{Acknowledgement}

This work was supported by grants from the Oswaldo Cruz Foundation (09224-7, PDTIS). 


\section{References}

Adler, B., Faine, S., 1977. Host immunological mechanisms in the resistance of mice to leptospiral infections. Infect. Immun. 17, 67-72.

Brown, C.R., Reiner, S.L., 1999. Experimental lyme arthritis in the absence of interleukin-4 or gamma interferon. Infect. Immun. 67, 3329-3333.

Brown, R.A., Blumerman, S., Gay, C., Bolin, C., Duby, R., Baldwin, C.L., 2003. Comparison of three different leptospiral vaccines for induction of a type 1 immune response to Leptospira borgpetersenii serovar Hardjo. Vaccine 21, 4448-4458.

Faine, S., 1962. The growth of Leptospira australis B in the kidneys of mice in the incipient experimental carrier state. J. Hyg. Camb. 60, 435-442.

Faine, S., 1965. Silver staining of spirochaetes in single tissue sections. J. Clin. Pathol. 18, 381-382.

Ko, A.I., Galvao Reis, M., Ribeiro Dourado, C.M., Johnson Jr., W.D., Riley, L.W., 1999. Urban epidemic of severe leptospirosis in Brazil. Salvador Leptospirosis Study Group. Lancet 354, 820-825.

Koizumi, N., Watanabe, H., 2004. Leptospiral immunoglobulin-like proteins elicit protective immunity. Vaccine 22, 1545-1552.

McBride, A.J., Athanazio, D.A., Reis, M.G., Ko, A.I., 2005. Leptospirosis. Curr. Opin. Infect. Dis. 18, 376-386.

Naiman, B.M., Blumerman, S., Alt, D., Bolin, C.A., Brown, R., Zuerner, R., Baldwin, C.L., 2002. Evaluation of type 1 immune response in naive and vaccinated animals following challenge with Leptospira borgpetersenii serovar Hardjo: involvement of $\mathrm{WC1}(+)$ gammadelta and CD4 T cells. Infect. Immun. 70, 6147-6157.

Nally, J.E., Chow, E., Fishbein, M.C., Blanco, D.R., Lovett, M.A., 2005a. Changes in lipopolysaccharide $\mathrm{O}$ antigen distinguish acute versus chronic Leptospira interrogans infections. Infect. Immun. 73, 3251-3260.

Nally, J.E., Fishbein, M.C., Blanco, D.R., Lovett, M.A., 2005b. Lethal infection of $\mathrm{C} 3 \mathrm{H} / \mathrm{HeJ}$ and $\mathrm{C} 3 \mathrm{H} / \mathrm{SCID}$ mice with an isolate of Leptospira interrogans serovar copenhageni. Infect. Immun. 73, 7014-7017.
Pereira, M.M., Andrade, J., Marchevsky, R.S., Ribeiro dos Santos, R., 1998 Morphological characterization of lung and kidney lesions in $\mathrm{C} 3 \mathrm{H} / \mathrm{HeJ}$ mice infected with Leptospira interrogans serovar icterohaemorrhagiae: defect of CD4+ and CD8+ T-cells are prognosticators of the disease progression. Exp. Toxicol. Pathol. 50, 191-198.

Rathinam, S.R., 2002. Ocular leptospirosis. Curr. Opin. Ophthalmol. 13, 381-386

Satoskar, A.R., Elizondo, J., Monteforte, G.M., Stamm, L.M., Bluethmann, H. Katavolos, P., Telford, S.R., 2000. Interleukin-4-deficient BALB/c mice develop an enhanced Th1-like response but control cardiac inflammation following Borrelia burgdorferi infection. FEMS Microbiol. Lett. 183, 319-325

Silva, E.F., Medeiros, M.A., McBride, A.J., Matsunaga, J., Esteves, G.S., Ramos, J.G., Santos, C.S., Croda, J., Homma, A., Dellagostin, O.A., Haake, D.A., Reis, M.G., Ko, A.I., 2007. The terminal portion of leptospiral immunoglobulin-like protein $\mathrm{LigA}$ confers protective immunity against lethal infection in the hamster model of leptospirosis. Vaccine 25 , 6277-6286.

Soares, M.B., Silva-Mota, K.N., Lima, R.S., Bellintani, M.C., Pontes-deCarvalho, L., Ribeiro-dos-Santos, R., 2001. Modulation of chagasic cardiomyopathy by interleukin-4: dissociation between inflammation and tissue parasitism. Am. J. Pathol. 159, 703-709.

Viriyakosol, S., Matthias, M.A., Swancutt, M.A., Kirkland, T.N., Vinetz, J.M. 2006. Toll-like receptor 4 protects against lethal Leptospira interrogans serovar Icterohaemorrhagiae infection and contributes to in vivo control of leptospiral burden. Infect. Immun. 74, 887-895.

Werts, C., Tapping, R.I., Mathison, J.C., Chuang, T.-H., Kravchenko, V., Saint G.I., Haake, D.A., Godowski, P.J., Hayashi, F., Ozinsky, A., Underhill, D.M., Kirschning, C.J., Wagner, H., Aderem, A., Tobias, P.S., Ulevitch, R.J., 2001. Leptospiral lipopolysaccharide activates cells through a TLR2-dependent mechanism. Nat. Immunol. 2, 346-352. 